\title{
Evaluación del desarrollo de la agricultura periurbana y propuesta de gestión integral en el distrito de Cayma, Arequipa, Perú
}

\author{
Evaluation of the development of peri-urban agriculture and integral management \\ proposal in the district of Cayma, Arequipa, Peru
}

Claudia Benavente Cárdenas ${ }^{1 *}$, Patricia Camargo Salcedo ${ }^{1}$, Guido Sarmiento Sarmiento ${ }^{1}$, Laydy Mena Chacón ${ }^{1}$

\begin{abstract}
RESUMEN
La agricultura periurbana (AP) es una actividad multifuncional que contribuye al desarrollo de políticas inclusivas e integrales con beneficios sociales, económicos y ambientales. La apertura comercial representa amenazas y oportunidades en el sector agropecuario de zonas periurbanas de nuestro país, transformándolo en un sector vulnerable, precario y sin estímulo. Este contexto requiere su intervención para convertirlo en un sector competitivo, moderno y sostenible.

El objetivo de este estudio fue determinar la capacidad técnico - productiva, estructura organizacional y el entorno externo de la actividad agrícola periurbana y plantear una propuesta de gestión de desarrollo agrícola con el gobierno local (GL). La investigación fue descriptiva - analítica, con enfoque cualitativo, método de investigación acción del tipo participativo. Los instrumentos de recolección de información fueron encuestas, observación participante, árbol de problemas, análisis FODA (fortalezas-oportunidadesdebilidades-amenazas) y grupos focales.

El estudio se inició con el diagnóstico del ambiente productivo, ambiental, político y social, permitiendo establecer causas reales de la problemática actual, potencialidades existentes y la relación e interacción con los demás factores del entorno de la actividad. Los resultados indican que el 43,5\% de agricultores se dedican a otras actividades económicas y el 40,7\% tiene menos de una hectárea. Los desafios que presenta la AP están relacionados con la ausencia de políticas públicas específicas, falta de información actual, escasa participación ciudadana, escasa intervención pública y privada en el sector agrícola periurbano. Luego se desarrolló participativamente la visión con los cambios que se pretende lograr. Se formularon estrategias de desarrollo integral con sus componentes organizacional, productivo - tecnológico, ambiental - recursos naturales y nutricional e higiene de alimentos. Finalmente se plantea que la agricultura en el distrito de Cayma se sustente en la cogestión entre agricultores y autoridades para su desarrollo integral.
\end{abstract}

Palabras clave: Pequeña agricultura, políticas integrales, desarrollo agrario

\begin{abstract}
Peri-urban agriculture (PA) is a multifunctional activity that contributes to the development of inclusive and comprehensive policies with social, economic and environmental benefits. The commercial opening represents threats and opportunities in the agricultural sector of peri-urban areas of our country, transforming it into a vulnerable sector, precarious and without stimulus; this context requires his intervention to turn it into a competitive, modern and sustainable sector.

The objective was to determine the technical-productive capacity, organizational structure and the external environment of the peri-urban agricultural activity and propound a management proposal for agricultural development with the local government $(L G)$. The research was a descriptive - analytical type, with a qualitative approach, an action research method of the participatory type; the instruments for gathering information were: surveys, participant observation, problem tree, SWOT analysis (strengthsweakness-opportunities-threats) and focus groups.

The study began with the diagnosis of the productive, environmental, political and social environment; allowing to establish real causes of the current problem, existing potentialities and the relationship and interaction with the other factors of the environment of the activity. The results indicate that $43.5 \%$ of farmers are engaged in other economic activities and $40.7 \%$ have less than one hectare; the challenges presented by the PA are related to the absence of specific public politics, lack of current information, scarce citizen participation, scarce public and private intervention in the peri-urban agricultural sector; then, the vision was developed participatively with the changes that are intended to be achieved; integral development strategies were formulated that have as components: the organizational, productive - technological, environmental - natural and nutritional resources and food hygiene. Finally, it is proposed that the development of agriculture in the Cayma district be based on co-management between farmers and authorities for their integral development.
\end{abstract}

Keywords: Small agriculture, comprehensive policies, agrarian development

1 Facultad de Agronomía. Universidad Nacional de San Agustín de Arequipa. Urb. Aurora s/n, Cercado. Arequipa, Perú.

* Autor para correspondencia: benavente.c22@gmail.com; gsarmientos@unsa.edu.pe

Fecha de recepción: 07 mayo, 2018.

Fecha de aceptación: 10 agosto, 2018.

DOI: http://dx.doi.org/10.4067/S0718-34292018005001101. Publicado en línea: 21-noviembre-2018. 


\section{Introducción}

La agricultura en el Perú es una actividad milenaria con importantes cambios en las últimas décadas (Eguren, 2009). Actualmente es una actividad productiva proveniente de múltiples cambios estructurales, logrando diversos tipos de agricultura. Uno de ellos es la AP, caracterizada por ser una actividad multifuncional con diversos componentes que permiten ofrecer respuesta a problemas sociales, económicos y ambientales. Desde 1980 fue considerada como fuente indispensable de alimentación para personas con mayores carencias alimentarias e instrumento para una alimentación más saludable en ambiente menos contaminado (FAO - IPES, 2016). Barsky (2012) define la AP como "una de las manifestaciones paisajísticas y sociales más características del borde periurbano, es el tipo particular de agricultura que en él se practica: el entramado de explotaciones primario intensivas".

La agricultura peruana constituye una economía donde el 85\% de los agricultores tienen parcelas con menos de 10 hectáreas, predominando unidades productivas con un área entre 3 y 10 hectáreas (33\%) (MINAGRI, 2017). En general, se estima que en Perú existen 2,3 millones de hogares cuya actividad principal es la agricultura (Zegarra y Tuesta, 2009), los cuales representan el 34\% de hogares peruanos y generan aproximadamente el 7,6\% del PBI Nacional (INEI, 2016). Excluyendo a Lima, la agricultura ocupa aproximadamente el $40 \%$ de la población económicamente activa - PEA (la sierra alcanza el 55\%) y representa entre el $20 \%$ y el $50 \%$ de los PBI regionales (Zegarra y Tuesta, 2009). Este gran dinamismo de la agricultura en los últimos años fue impulsado por el importante crecimiento de las exportaciones y el aumento en la producción agrícola y pecuaria (MINAG, 2012).

La actividad agrícola en estudio se desarrolla en el contexto periurbano del distrito de Cayma, región Arequipa - Perú. Este es uno de los distritos más tradicionales de Arequipa, ubicado a una altitud de 2.403 m.s.n.m., extensión territorial de 246,31 km², población total de 91.802 habitantes (INEI, 2016), con un sector agropecuario caracterizado por estar orientado a producir más alimentos para el sustento de la población regional y de autoconsumo. AGAP (2015) indica que las unidades agroproductivas corresponden a una pequeña agricultura que atiende exclusivamente el mercado interno, situación que es materia de análisis, ya que si bien los indicadores de crecimiento de la agricultura en el país se han elevado considerablemente estos últimos 10 años, no corresponderían a las características de la pequeña agricultura como la que representa el distrito. Más bien esta situación se atribuiría al "boom agroexportador" promovido por la explotación de grandes extensiones de tierra en manos de grandes grupos económicos. El mismo autor afirma que la producción de productos agrícolas para exportación creció a una tasa de 19\% anual y la producción agrícola moderna en general, a una tasa de $8 \%$ en los últimos diez años.

Se considera como causa de la situación actual de esta actividad el limitado interés en el desarrollo de investigación que permita establecer el origen real de los problemas existentes y sus relaciones e interacción con los factores del entorno interno y externo. Este contexto motivó el objetivo de la presente investigación: evaluar el desarrollo de la AP, determinando la capacidad técnico-productiva, estructura organizacional, el entorno externo de la actividad, y plantear una propuesta de gestión integral para el desarrollo de la agricultura a nivel de GL.

\section{Materiales y métodos}

La investigación fue descriptiva - analítica, con enfoque cualitativo, empleando el método investigación acción del tipo participativo. Tuvo como soporte la descripción y análisis de los actores vinculados a la gestión municipal y al desarrollo local del borde periurbano del distrito de Cayma. El proceso fue eminentemente participativo, convocando a los distintos sectores del área para recoger sus conocimientos sobre los recursos del sector, siendo los grupos de interés, agricultores representados por la Comisión de Usuarios de Acequia Alta de Cayma, Cerro Colorado y Anexos - CUACA; Municipalidad Distrital de Cayma - MDC; Junta de usuarios del chili regulado - JUCR; y, como ente público encargado de investigar y vincular resultados, la Universidad Nacional de San Agustín de Arequipa - UNSA. 
La investigación se desarrolló en tres fases desde julio 2016 hasta junio 2017. La primera fase consistió en la revisión documental mediante consulta, lectura y análisis de bibliografía, que permitió adquirir bases teóricas y una visión general de la problemática que presenta la AP en nuestro país. Se utilizaron libros, revistas, artículos, planes de desarrollo a nivel local, regional y nacional, perfiles y antecedentes de proyectos realizados. Simultáneamente se hicieron visitas de campo para identificar la zona y establecer contacto con representantes de los grupos de interés. En la segunda fase se realizaron encuestas semiestructuradas a 108 agricultores, reunión con expertos y grupos focales. Se inició con la JUCR, luego con agricultores y representantes de la MDC y la UNSA, con el fin de escuchar las distintas opiniones de los representantes de cada comisión a nivel regional y evaluar la problemática de la agricultura de Cayma, así como la participación del GL, nacional y entidades públicas relacionadas con la actividad. En la tercera fase, toda la información obtenida se estructuró, ordenó y organizó, para ser evaluada y analizada. En esta fase se realizó la triangulación de los instrumentos y técnicas empleadas (análisis FODA, matriz defensiva y ofensiva, árbol de problemas, entre otros), analizando la totalidad del significado de la información obtenida aplicada al desarrollo local y descomponiéndola en sus partes integrantes. El planteamiento preliminar de la propuesta integral para el sector agrícola del distrito de Cayma buscó la participación del GL en función de los programas de desarrollo agrícola establecidos a nivel nacional.

Para la determinación del tamaño de la muestra (cantidad de agricultores a encuestar) se utilizó la siguiente fórmula (Hernández, 1979):

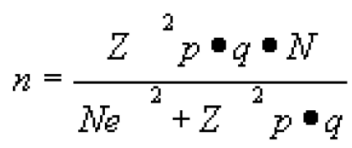

Donde:

- $\quad \mathrm{N}=$ Total de la población (717 agricultores miembros de la CUACA)

- $\mathrm{Z}=2,58$ al cuadrado (si la seguridad es del 99\%)

- $\mathrm{p}=$ proporción esperada (en este caso $5 \%=0,05$ )

- $\quad \mathrm{q}=1-\mathrm{p}$ (en este caso $1-0,05=0,95)$

- $\mathrm{d}=$ precisión $5 \%$

\section{Resultados y discusión}

\section{Características de la zona de estudio}

El área de estudio se encuentra en la periferia de la ciudad de Arequipa, clasificada como zona periurbana. La cercanía con la ciudad y el acceso son elementos importantes para la comercialización e implementación de actividades agrícolas. Asimismo, la calidad del agua para regadío es reconocida por sus bajos niveles de contaminación, constituyéndose en una ventaja competitiva.

La topografía accidentada de Cayma hace posible la existencia de diversos microclimas, predominando un ambiente árido seco, con temperaturas que oscilan entre 0 y $30^{\circ} \mathrm{C}$, pendiente media $4,6 \%$, precipitaciones estacionales entre 72 - $105 \mathrm{~mm}$ anuales y radiación promedio anual de 8,7 horas sol por día (MDC, 2006). Estas características edafoclimáticas permiten la explotación continua de diversos cultivos hortícolas y forrajeros.

\section{Caracterización del sistema productivo}

La actividad principal es la agricultura y en bajo porcentaje la ganadería y crianza de animales menores (Tabla 1). El 43,5\% de agricultores se dedican a otras actividades económicas desarrolladas dentro de la zona y en sectores colindantes, debido principalmente a que solo el $11,1 \%$ califica su situación económica como buena. Las expectativas de mejora son muy bajas, en desmedro de la AP como una forma de vida, percibiéndola como una fuente secundaria de ingresos para los agricultores, en especial para aquellos que poseen menor extensión de tierras (Tabla 1).

La influencia de grandes fundos en la producción agrícola, la crisis económica nacional e internacional, las políticas agrarias e incorporación de nuevas tierras agrícolas de grandes extensiones (irrigaciones) fueron cambiando la estructura productiva del sector primario, para convertirlo en un sector con limitada competitividad en el mercado, y por lo tanto no rentable.

Del mismo modo, la continua urbanización de las zonas periurbanas pone en peligro la preservación de las zonas rurales. Aun existiendo las ordenanzas municipales, estas son desconocidas o simplemente 
Tabla 1. Características de la actividad agrícola y manejo de producción periurbana de Cayma

\begin{tabular}{|c|c|}
\hline Categoría & Resultado de encuesta a agricultores del distrito de Cayma \\
\hline Explotación & $73,1 \%$ solo agricultura; $26,9 \%$ agricultura y ganadería. \\
\hline Propiedad & $\begin{array}{l}19,4 \% \text { por compra; } 38,9 \% \text { por herencia; } 38,9 \% \text { arrendatarios; } \\
2,8 \% \text { por asociación. }\end{array}$ \\
\hline Motivación a la actividad & $\begin{array}{l}58,8 \% \text { fuente principal de ingresos; } 31,5 \% \text { fuente extra de } \\
\text { ingresos; } 9,3 \% \text { autoconsumo; } 0,9 \% \text { otros motivos. }\end{array}$ \\
\hline Tamaño de predio & $40,7 \%<1$ ha; $2,8 \%=<4$ ha; $0,9 \%>5$ ha. \\
\hline Semilla & $66,5 \%$ usa semilla certificada; $23,1 \%$ común; $10,3 \%$ propia. \\
\hline Riego & $98,1 \%$ por gravedad; $1,9 \%$ a goteo y aspersión. \\
\hline Fertilización & $5,6 \%$ con abonos orgánicos; $8,3 \%$ químicos; $86,1 \%$ mixtos. \\
\hline Agroquímicos & $\begin{array}{l}8,3 \% \text { aplica agroquímicos permitidos. } 42,6 \% \text { no identifica cuáles } \\
\text { son los insumos autorizados por SENASA }{ }^{1} \text {. }\end{array}$ \\
\hline Maquinaria & $53,7 \%$ emplea maquinaria agrícola; $16,7 \%$ tracción animal. \\
\hline $\mathrm{MA}^{2}$ & $\begin{array}{l}70,4 \% \text { su actividad es amigable con el MA; } 76,6 \% \text { utiliza } \\
\text { adecuadamente el recurso suelo, agua y vegetal. }\end{array}$ \\
\hline Destino de cosecha & $80,6 \%$ a mayoristas e intermediarios; $19,4 \%$ venta directa. \\
\hline
\end{tabular}

${ }^{1}$ Servicio Nacional de Sanidad Agraria del Perú. ${ }^{2}$ Medio Ambiente.

ignoradas (Tabla 2). Es prioritaria la intervención de las autoridades a fin de informar, prevenir, monitorear, controlar y sancionar a aquellas personas que incumplan disposiciones normativas. De esta manera será posible proteger las zonas rurales periurbanas y mantener un equilibrio ambiental.

Tabla 2. Percepción de los elementos de gestión e intervención pública en el sector agrícola periurbano de Cayma

\begin{tabular}{|c|c|}
\hline Categoría & Percepción de los agricultores de Cayma \\
\hline ¿Conoce el término $\mathbf{A P}{ }^{1}$ ? & $34,3 \%$ conoce o ha escuchado. \\
\hline ¿Conoce qué es un plan estratégico? & $28 \%$ conoce, $4 \%$ ha leído alguno. \\
\hline $\begin{array}{l}\text { ¿Conoce } \mathrm{OM}^{2} \text { que prohíben la venta } \\
\text { de predios rurales? }\end{array}$ & $39 \%$ conoce que la zona de campiña no puede ser urbanizada. \\
\hline Intervención del GN ${ }^{3}$ & $84 \%$ afirma sentirse "un sector abandonado". \\
\hline Intervención del GL ${ }^{4}$ & $\begin{array}{l}77,8 \% \text { ninguna; } 13,9 \% \text { infraestructura de riego; } 4,6 \% \text { vías de } \\
\text { comercialización; } 3,7 \% \text { capacitación técnica. }\end{array}$ \\
\hline $\begin{array}{l}\text { Responsabilidad del desarrollo de } \\
\text { la AP }\end{array}$ & $\begin{array}{l}24 \% \text { le atribuyen al MINAGRI }{ }^{5} \text { como representante del GN, } 20 \% \\
\text { GR }^{6}, 22 \% \text { GL; } 23 \% \text { los mismos agricultores. }\end{array}$ \\
\hline Acerca de la asociatividad & $\begin{array}{l}\text { 40\% conoce la importancia que tiene esta; } 48 \% \text { está dispuesto a } \\
\text { participar de una asociación de agricultores. }\end{array}$ \\
\hline Interés en capacitación & $\begin{array}{l}\text { 31,10\% participaría de charlas técnicas, el } 25,91 \% \text { en visitas } \\
\text { guiadas a parcelas o centros demostrativos. }\end{array}$ \\
\hline Fuente de asesoramiento & $\begin{array}{l}55,6 \% \text { de casas comerciales; } 3,7 \% \text { asesoramiento profesional; } \\
18,5 \% \text { no recibe; } 18,5 \% \text { no le interesa. }\end{array}$ \\
\hline
\end{tabular}

Los cultivos económicamente importantes son hortalizas y verduras, predominando brócoli (Brassica olereacea var. Itálica $\mathrm{L}$.) y arveja a (Pisum sativum L.), seguidos por repollo (Brassica oleracea var. Capitata L.), lechuga (Lactuca sativa L.), zapallo (Cucurbita maxima L.) y pepinillo (Cucumis sativus L.); y de menor importancia, habas (Vicia faba L.) y papa (Solanum tuberosum L.) (INEI, 2013). La productividad de estos cultivos es muy variable por la forma de conducción, extensión y nivel tecnológico de los propios agricultores. 
El principal activo que poseen los agricultores son sus tierras, por lo tanto sus derechos de propiedad deben estar bien definidos para lograr un reconocimiento legal. Sin embargo, existe heterogeneidad en la forma de tenencia de los predios agrícolas, al igual que en el tamaño de estos (Tabla 1), clasificado como de pequeña y mediana escala por el IV Censo Nacional Agropecuario (INEI, 2013). Este fraccionamiento de tierras en pequeños minifundios representa una limitante en la eficiencia productiva, al mismo tiempo que eleva los costos de producción, más aún si los propietarios son renuentes a la actualización de sus datos y nuevas tecnologías por temor o desconocimiento de su utilidad.

En cuanto a las características de los agricultores, se observó resistencia y baja predisposición al cambio, a pesar de que la mayoría de ellos cuentan con experiencia y conocen sus sistemas productivos. Este comportamiento se puede atribuir a la limitada sensibilización que los encamine a asumir su responsabilidad con el medio ambiente y a la forma de manejo de los recursos naturales, ya que consideran que su actividad es sostenible y no altera el agroecosistema (Tabla 1). Este aspecto genera controversia y preocupación, debido a que existe un alto porcentaje de agricultores que no identifican agroquímicos prohibidos por el Servicio Nacional de Sanidad Agraria - SENASA (Tabla 1). Estos insumos son empleados a su criterio o por recetas de las empresas comerciales. Solo un sector reducido de agricultores reciben asesoría técnica gratuita en el uso seguro de agroquímicos (Tabla 2).

La comercialización de los productos se realiza principalmente mediante intermediarios $r$ (Tabla 1). La búsqueda de altos rendimientos acompañada de buenos precios es un criterio de alto valor para los agricultores, pero se ve afectada por la desigualdad comercial, ya que no tienen la capacidad de disminuir los costos de insumos agrícolas o aumentar los precios de venta de los productos. La causa es la ausencia de normativa en la comercialización, siendo el pequeño agricultor el más afectado.

\section{Estructura organizacional de la AP}

El Ministerio de Agricultura y Riego - MINAGRI - es la entidad estatal responsable de la actividad agrícola en el Perú. Interviene mediante programas y los diversos organismos adscritos. Uno de ellos es la Autoridad Nacional del Agua - ANA, que mediante la Autoridad Administrativa del Agua - AAA y las
Autoridades Locales del Agua - ALA, Juntas y Comisiones de Usuarios, se encarga de administrar, conservar y proteger los recursos hídricos en las cuencas para alcanzar su aprovechamiento sostenible (ANA, 2017).

Los agricultores evaluados son miembros de la CUACA, que cuenta con 659 ha de predios y 1,071 agricultores inscritos en su padrón. No obstante, estos no son solo agricultores, sino que también están incluidas instituciones privadas, colegios, parques y áreas que ya fueron urbanizadas, quedando 717 agricultores, de los cuales menos del $20 \%$ tienen sus datos actualizados e incluso algunos fallecieron. $\mathrm{La}$ infraestructura de riego se encuentra dividida en 7 sectores: Cayma, Challpampa, Chilina, Carmen Alto, Acequia Alta y La Tomilla. La CUACA no cuenta con un plan estratégico, siendo este un instrumento muy importante y base de su organización que establece las acciones que se realizarán a fin de cumplir los objetivos en común para su desarrollo (Tabla 2).

Los desafíos que presenta la AP en Cayma están relacionados con la falta de prioridad en las políticas públicas específicas para este sector, que deriva de la escasa presencia del Estado y las diferentes instituciones responsables del agro, como SENASA, Instituto Nacional de Información Agraria - INIA y Gobiernos Regionales - GR, que por medio de sus Agencias Agrarias deben pretender el desarrollo agrícola.

La globalización y la intensa apertura comercial en nuestro país representan tanto amenazas como oportunidades para el sector agropecuario de las zonas periurbanas, siendo un sector precario, vulnerable y sin estímulo. Por este motivo se hace necesario que este sector agrícola tradicional sea competitivo, moderno y sostenible. Esta transformación requiere de grandes esfuerzos tanto del sector público como del privado y debe empezar en materia de información y comunicación, para articular cada uno de los actores involucrados, comenzando por el MINAGRI como entidad responsable del desarrollo de la actividad, hasta llegar a los agricultores, para tomar decisiones e integrar el sector en los procesos productivos, comerciales y de servicios.

Otro factor es la falta de información en el sector agrario. El agricultor no cuenta con datos reales, actualizados y permanentes de precios, nuevas tecnologías, oportunidades de mercado local, nacional e internacional. No existe un sistema informativo que permita al agricultor tomar decisiones apropiadas en la planificación, producción, y conocimientos, a pesar 
de que hay organismos que brindan información como la agencia agraria, el Sistema de Información Agraria - SIA - y el INEI. Sin embargo, los datos que ofrecen están dispersos y desorganizados, siendo complicado su acceso y comprensión.

\section{Entorno externo de la AP en Cayma}

Desde hace unas décadas, más GL y GN han implementado programas y políticas de agricultura urbana y AP a nivel nacional e internacional, como es el caso de El Alto (Bolivia) con los microhuertos familiares que benefician a más de 500 familias pobres urbanas. En Bogotá, Medellín y Cartagena (Colombia) los gobiernos y la cooperación internacional han capacitado a más de 50 mil personas para realizar huertas en diversos espacios urbanos y periurbanos. En Curitiba (Brasil) unos $8 \mathrm{mil}$ agricultores urbanos y 6 mil escolares cultivan alimentos en 1.280 hectáreas. De igual manera, Belo Horizonte (Brasil) ha integrado la agricultura urbana en su política de seguridad alimentaria y en el ordenamiento territorial (FAO, 2016).

La AP en nuestro país es considerada de pequeña escala y se le atribuye la responsabilidad de la seguridad alimentaria interna. Las denominaciones que se le dan son, entre otras, "agricultura de campiña" y "agricultura familiar".

En el Perú, la Municipalidad de Lima Metropolitana (2012) incorporó, mediante la Ordenanza N ${ }^{\circ} 1629$, un programa que propone y gestiona proyectos de inversión pública orientada a desarrollar actividades agrícolas dentro de la ciudad y en las periferias, como una alternativa de empleo y autoabastecimiento de alimentos de las familias. No obstante, a nivel local la intervención pública y privada en el sector agrícola periurbano está ausente, los agricultores se sienten olvidados por los 3 niveles de gobierno, la burocracia que existe y la falta de interés se ven reflejadas en el único proyecto en proceso que tiene la CUACA en el sector riego: Proyecto Sub-sectorial de Irrigación. Es necesario destacar la iniciativa de la MDC y la UNSA para firmar el convenio junto a la CUACA con el fin de intervenir en este sector. Otro programa que es incentivado por la MDC y la CUACA es la feria "De la chacra a la olla", pero que lamentablemente no está dirigida adecuadamente, y esto se refleja en la escasa participación de agricultores (5\%) debido a que solo un pequeño porcentaje de su producción se vendería allí. Además tendrían que invertir tiempo para poder vender de forma directa, razón por la cual prefieren ofrecer sus productos a los intermediarios (Tabla 1).
La MDC afirma tener un rol promotor y de gestión, pero que no percibe interés por parte de los agricultores. Si bien se requiere mayor participación de la ciudadanía, tampoco se realizan acciones para propiciarla, creando así un ambiente desfavorable entre el agricultor y los representantes de las entidades públicas. Por otra parte, resalta la persistencia de algunos agricultores, liderados por su junta directiva, para ser escuchados, para ser partícipes de un cambio, para que los tomen en cuenta en las decisiones y proyectos del municipio, y que la municipalidad, por su parte, encuentre metodologías más adecuadas que permitan y generen mayor participación ciudadana.

Diversas experiencias, como el caso del Programa Nacional de Agricultura Urbana (Cuba), impulsaron la actividad agrícola periurbana a escala nacional, provincial y municipal (FAO, 2016), y demuestran que cuando los gobiernos locales gestionan la producción intensiva de alimentos, se generan mejores condiciones para la sostenibilidad de los pobladores. Para ello es necesaria la voluntad política de los gobiernos y una capacitación permanente para la sustentabilidad y socialización de la AP.

\section{Propuesta de gestión integral}

A fin de elaborar la propuesta de gestión integral, se definió el problema central (Figura 1) mediante la vinculación y análisis de los datos obtenidos en las dos primeras fases del estudio.

Se determinó como objetivo central el "Desarrollo de la AP en el distrito de Cayma mediante la cogestión entre agricultores y autoridades comprometidas con el agro". En la propuesta se consideraron los componentes productivos - tecnológicos, organizacionales, ambientales - recursos naturales y nutrición e higiene de alimentos. En la Tabla 3 se presenta la propuesta de articulación de objetivos de gestión integral en la actividad agrícola con los objetivos del GL.

Para el cumplimiento de los Objetivos Estratégicos Propuestos en la Tabla 3, se consideran las siguientes Estrategias Operativas - EO:

- EO1: Promover el desarrollo empresarial a partir de la cooperación público- privada, mediante:

- Promoción de redes y mesas de concertación.

- Promoción y priorización de emprendimientos no tradicionales (turismo, industrias culturales).

- Actividades de capacitación y/o asistencia técnica. 


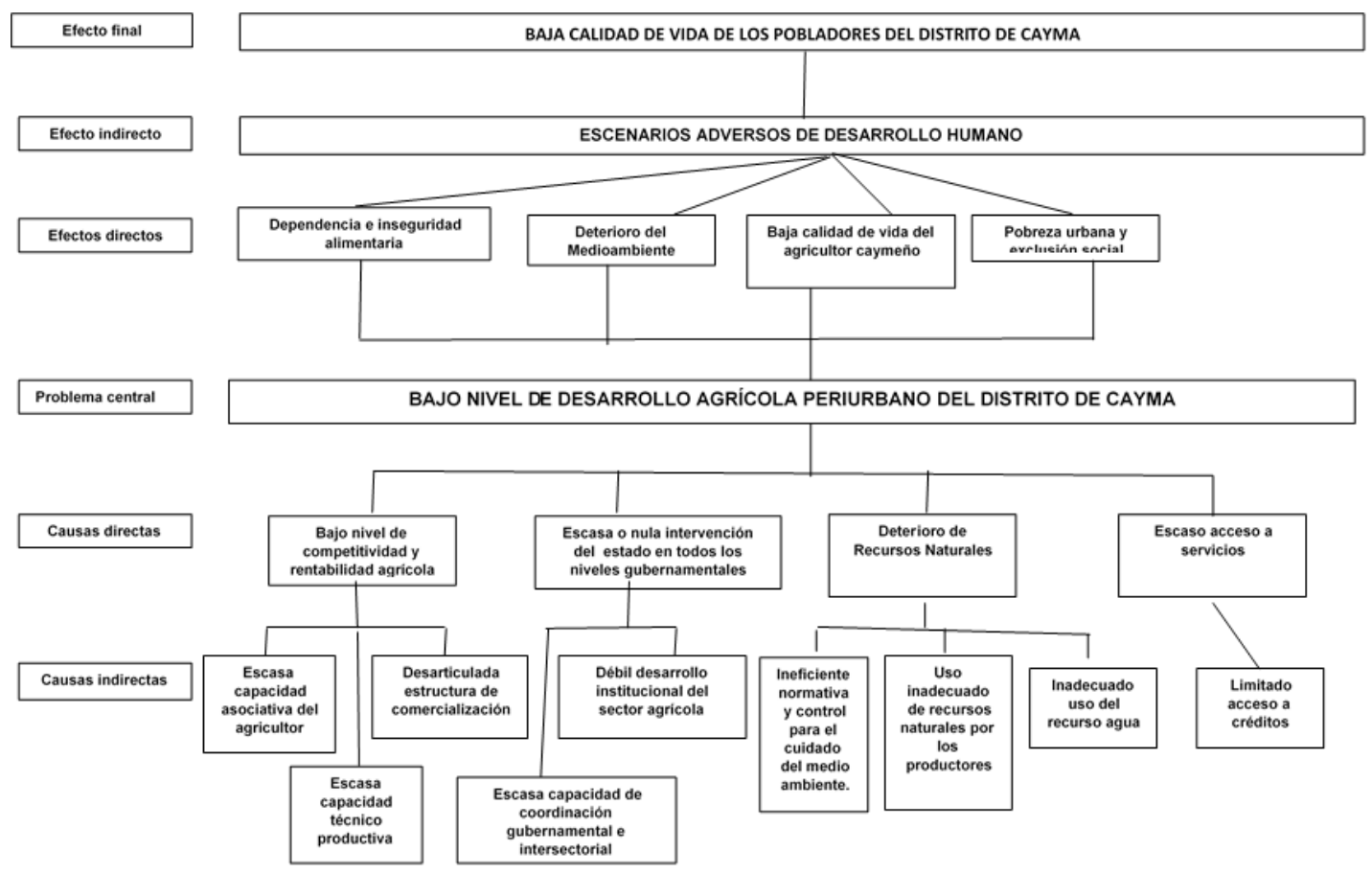

Figura 1. Árbol de problemas de la actividad agrícola periurbana en Cayma

Tabla 3. Articulación de objetivos de la propuesta de gestión integral con el Plan de Desarrollo concertado de la Municipalidad Distrital de Cayma.

\begin{tabular}{|c|c|c|}
\hline \multirow{2}{*}{ Objetivo estratégico de la propuesta } & \multicolumn{2}{|c|}{$\begin{array}{c}\text { Plan de desarrollo concertado de la Municipalidad } \\
\text { Distrital de Cayma* }\end{array}$} \\
\hline & Objetivos & Lineamientos \\
\hline $\begin{array}{l}\text { Fortalecer la organización de los } \\
\text { agricultores para una adecuada gestión de } \\
\text { espacios productivos y alianzas estratégicas }\end{array}$ & \multirow{2}{*}{$\begin{array}{l}\text { Incrementar las } \\
\text { oportunidades de } \\
\text { empleo e ingresos } \\
\text { adecuados }[\ldots]\end{array}$} & $\begin{array}{l}\text { Promover la formalización, } \\
\text { asociatividad, competitividad y } \\
\text { articulación a mercado }[\ldots]\end{array}$ \\
\hline $\begin{array}{l}\text { Incrementar de manera sostenible la } \\
\text { producción y mejorar su inocuidad y } \\
\text { calidad }\end{array}$ & & $\begin{array}{c}\text { Fomentar las alianzas público } \\
\text { - privadas para la inversión } \\
\text { descentralizada en proyectos } \\
\text { estratégicos }[\ldots]\end{array}$ \\
\hline $\begin{array}{c}\text { Mitigar el impacto ambiental de la } \\
\text { actividad agrícola a través del uso racional } \\
\text { de los recursos }\end{array}$ & \multirow{2}{*}{$\begin{array}{l}\text { Incrementar el } \\
\text { bienestar y felicidad } \\
\text { de las familias }[\ldots]\end{array}$} & $\begin{array}{l}\text { Mejorar la calidad ambiental para } \\
\text { las familias del distrito [...] en un } \\
\text { contexto del cambio climático. }\end{array}$ \\
\hline $\begin{array}{l}\text { Producción de alimentos con un enfoque en } \\
\text { seguridad alimentaria y nutricional }\end{array}$ & & $\begin{array}{c}\text { Constituir participativamente }[\ldots] \\
\text { para el desarrollo de la población } \\
\text { del distrito. }\end{array}$ \\
\hline
\end{tabular}

*MDC, 2015. ${ }^{1}$ Reserva Nacional de Salinas y Aguada Blanca. 
- EO2: Facilitar información útil para el desarrollo empresarial y la atracción de inversiones, a través de:

- Constitución y formalización de empresas.

- Promoción de la ofertade servicios financieros.

- Promoción de la oferta de servicios de desarrollo empresarial (capacitaciones, asistencias técnicas, entre otros).

- Identificación de mercados y oportunidades de negocios.

- Utilización de los instrumentos de gestión urbana (Plan Director y Catastro), como fuentes de información y orientación.

- EO3: Fomentar la formalización empresarial y la eliminación de barreras de acceso al mercado:

- Apoyar la asociatividad y el fortalecimiento gremial mediante la constitución de redes y asociaciones empresariales rurales; así como la promoción de foros, mesas de diálogo y concertación sobre problemas entre los asociados.

\section{Conclusiones}

El análisis de los componentes técnico - productivo, organizacional y los factores externos que determinan la actividad agrícola en el distrito de Cayma permitió precisar y validar el problema planteado en la investigación, como es el escaso desarrollo de la AP. La causa y consecuencia de esta situación es principalmente el insuficiente nivel técnico de conducción (calificado como bajo a medio), siendo necesaria y prioritaria la creación de políticas diferenciadas y específicas para enfrentar los problemas estructurales y de organización, como la extrema fragmentación de tierras, deficiente asistencia técnica, mal uso de los recursos naturales, alto riesgo productivo, defectuosa estructura de la cadena comercial y falta de una plataforma de servicios agropecuarios para atender eficientemente a pequeños productores mediante metodologías que impulsen y desarrollen todos los espacios de participación y concertación entre agricultores y autoridades.

Por parte de la MDC existe acercamiento con el sector agrícola, lo que posibilita y facilita la atención futura a la AP; sin embargo, no se tiene o se desconoce el soporte que articule objetivos administrativos y operativos. Asimismo, su estructura organizacional no es adecuada, y si bien existe capacidad operativa, esta no responde a la visión, misión y objetivos institucionales. Se demuestra la necesidad de capacitar a los profesionales y la posibilidad de integrar a otros actores para la atención requerida. Existe el convencimiento de que la municipalidad debe integrar a los productores en sus diferentes planes y se evidencia predisposición para incorporar normas específicas a favor de su inclusión.

La vinculación de las universidades y los centros de investigación, desarrollo y transferencia tecnológica permitiría concertar aspectos académicos y productivos. Actualmente las labores de los investigadores son diferentes a la agenda de los políticos, y ambos son diferentes a los aspectos productivos. Se debe procurar atender este tema para lograr la integración e interrelación entre estos actores.

La estructura social de la CUACA cuenta con la participación de los agricultores; sin embargo, estos no se sienten empoderados de la pertenencia a su organización. La falta de liderazgo, débil cultura corporativa, desconocimiento de liderazgo y de trabajo en equipo en esta organización se considera como su principal debilidad.

Se concluye que el bajo desarrollo de la actividad agrícola periurbana en el distrito de Cayma se debe a la deficiente interacción de diversos factores técnicos y sociales, tales como bajo nivel de competitividad y rentabilidad agrícola, escasa o nula intervención del Estado en todos sus niveles, uso inadecuado de recursos y escaso acceso a los servicios. A partir de estos factores identificados se estructuraron estrategias de gestión integral municipal para el desarrollo de la AP, siendo el inicio para generar alternativas de solución a la problemática de la actividad en esta zona.

\section{Agradecimientos}

A la Universidad Nacional de San Agustín de Arequipa - Perú por el financiamiento otorgado para ejecutar esta investigación. 


\section{Literatura citada}

ANA (Autoridad Nacional de Agua, Perú).

2016. Proceso de adecuación de las organizaciones de usuarios de agua a la Ley 30157 y su reglamento. Lima, Perú. 161 p.

Barsky, A.

2012. La complejidad territorial de la interfase urbano-rural como soporte para el desarrollo de la agricultura periurbana. En: Mitidieri, M. y Corbino G. (ed). Manual de horticultura periurbana. INTA. Buenos Aires, Argentina. 2012. pp 23 - 28.

Eguren, F.

2009. Reforma Agraria y desarrollo rural en el Perú. CEPES, Lima, Perú. 21 p.

\section{FAO-IPES}

2016. Agricultura urbana y periurbana en América Latina y el Caribe: una realidad. Organización de las Naciones Unidas para la Agricultura y la Alimentación - IPES Promoción FAO del Desarrollo Sostenible. 3 p.

2016. Agricultura urbana y periurbana en América Latina y el Caribe: Compendio de casos. Organización de las Naciones Unidas para la Agricultura y la Alimentación. Roma, Italia. 295 p. Hernández, $\mathrm{O}$.

1979. Elementos de probabilidad y estadística. Fondo de Cultura Económica. México. 355 p.

INEI (Instituto Nacional de Estadística e Información, Perú). 2013. Resultados definitivos: IV Censo Nacional Agropecuario 2012. Ministerio de Agricultura y Riego del Perú. Lima, Perú. 63 p.

INEI (Instituto Nacional de Estadística e Información, Perú). 2016. Perfil Socio demográfico del Perú. 2 ed. Lima, Perú. $474 \mathrm{p}$.
MDC

2006. Caracterización del Distrito de Cayma: Plan Urbano Distrital PUD 2006 - 2015. Municipalidad Distrital de Cayma. Arequipa, Perú. 63 p.

MDC

2015. Plan de Desarrollo Municipal Concertado del Distrito de Cayma al 2021. Municipalidad Distrital de Cayma, Perú. Arequipa, Perú. 155 p.

MINAG.

2012. Plan estratégico sectorial multianual 2012-2016. Ministerio de Agricultura, Perú. Lima, Perú. 123 p. MINAGRI.

2016. Plan Estratégico Institucional 2016-2018. Ministerio de Agricultura y Riego. Lima, Perú. 196 p.

MINAGRI.

2017. Problemas tipo de la agricultura peruana. Ministerio de Agricultura y Riego, Perú. Lima, Perú. 4 p.

Municipalidad de Lima Metropolitana.

2012. Ordenanza No 1629: Ordenanza Marco de promoción de la Agricultura Urbana como estrategia de gestión ambiental, seguridad alimentaria, inclusión social y desarrollo económico local de la provincia de Lima. Diario Oficial-El Peruano. Lima, Perú.

Zegarra, E. y Tuesta, J.

2009. Crecimiento agrícola, pobreza y desigualdad en el Perú rural. En: Graziano, J.; Gómez, S. y Castañeda, R. (ed). Boom Agrícola y Persistencia de la Pobreza Rural: Estudio de ocho casos. FAO. Roma, Italia. 299-329 p. 
